\title{
The Antiparkinsonian Actions and Pharmacokinetics of Transdermal (+ )-4-Propyl-9-Hydroxynaphthoxazine (+ PHNO): Preliminary Results
}

\author{
$*$ R. J. Coleman, $\nmid$ K. W. Lange, $* \dagger N$. P. Quinn, $\ddagger$ A. E. Loper, \\ $\ddagger$ J. V. Bondi, $\ddagger$ M. Hichens, ${ }^{*} \ddagger S$. M. Stahl, and $*$ C. D. Marsden \\ *The Institute of Neurology, †The Institute of Psychiatry, London, U.K. and $\ddagger$ Merck, \\ Sharp and Dohme Research Laboratories, Harlow, U.K. and Pennsylvania, U.S.A.
}

\begin{abstract}
Summary: (+)-4-Propyl-9-hydroxynaphthoxazine (+ PHNO) is a potent dopamine agonist that has been administered transdermally to four patients with Parkinson's disease and "on-off' fluctuations. Skin patches of increasing size were used to treat these patients, who also received infrequent doses of oral levodopa if required. The effect of + PHNO was measured as an increased duration of action of individual levodopa doses. The clinical effect measured in this way was directly proportional to the plasma concentrations of + PHNO achieved. The plasma concentrations of + PHNO began to rise $4-6 \mathrm{~h}$ after patch application and reached a steady state by $24 \mathrm{~h}$. The final plasma concentration of + PHNO was proportional to the area of skin covered. Key Words: Parkinson's disease-On-off fluctuations-Transdermal drug deliveryDopamine agonist-(+)-4-Propyl-9-hydroxynaphthoxazine.
\end{abstract}

For many patients with Parkinson's disease, the development of "on-off" fluctuations blights their otherwise good response to levodopa. This on-off syndrome consists of a variable and unpredictable response to regular oral levodopa and is now recognised as a major source of disability in patients with Parkinson's disease who are receiving chronic levodopa therapy. Someone with a fully developed on-off syndrome exhibits the following features: (a) "off' episodes characterised by typical parkinsonian rigidity, bradykinesia, and tremor (and sometimes dystonia, pain, panic, and depression) when levodopa fails to be effective; (b) "on" periods after a dose of levodopa during which there is a marked improvement in the features of parkinsonism but accompanied by peak-dose or diphasic choreiform, dystonic, or stereotyped involuntary movements that may themselves be

Address correspondence to Dr. R. J. Coleman at Department of Neurology, The London Hospital, Whitechapel, London E1 IBB, U.K. 
disabling; (c) a tendency to switch suddenly between "off" and "on" states in a way that cannot readily be predicted from the timing of individual levodopa doses or from the levels of levodopa in the plasma $(1,2)$.

It is known that such fluctuations can be significantly improved if plasma levodopa concentrations are kept constant, for example by means of an intravenous infusion of levodopa, and various attempts have been made to adapt this model of continuous dopaminergic stimulation for practical use. These have included the use of sustained-release levodopa tablets and trials using subcutaneous infusions of the dopamine agonists lisuride and apomorphine $(3,4)$.

(+)-4-Propyl-9-hydroxynaphthoxazine (+PHNO), a novel and highly potent dopamine agonist, has recently been shown to reverse the parkinsonian deficits produced by 1 -methyl-4-phenyl-1,2,3,6-tetrahydropyridine (MPTP) in marmosets and is effective given by oral and subcutaneous routes $(5,6)$. In Parkinson's disease, + PHNO is effective in both mildly affected patients with a short duration of illness, and in more severely affected patients with on-off fluctuations $(7,8)$. + PHNO would appear to be a good candidate for use in the control of on-off fluctuations if it could be administered in a continuous manner.

It has been shown that + PHNO applied to the skin of rats is absorbed and produces systemic pharmacological effects (9). + PHNO is soluble in both aqueous and lipid media and this is known to be an important physical property of drugs that are capable of transdermal absorption $(10,11)$. High potency, extensive first-pass metabolism, and the desire for continuous delivery are all features of + PHNO that make it an ideal candidate for this method of administration. In view of this, skin patches containing + PHNO have been designed and manufactured with the intention of creating a transdermal continuous drug delivery system. The necessary technology has already been used in the management of conditions in which continuous therapy is of benefit and for which the necessary drugs have suitable physical and pharmacological properties. Examples are nitroglycerin for angina, clonidine for hypertension, scopolamine for motion sickness, and oestrogens for postmenopausal hormone replacement therapy (12).

We have investigated the use of + PHNO skin patches in patients with Parkinson's disease who have developed on-off fluctuations. The purposes of the study were to demonstrate whether skin patches were capable of delivering + PHNO into the systemic circulation and to assess the pharmacokinetics of this absorption route.

\section{METHODS}

Four patients with idiopathic Parkinson's disease entered the study having given written informed consent. They were all levodopa-responsive but had developed on-off fluctuations in their motor response to regular oral medication. (Details of the patients are shown in Table 1.)

+ PHNO was administered by applying circular skin patches to the anterior chest wall. The patches were capable of delivering + PHNO at a rate substantially greater than the rate of skin penetration. Two sizes of patch were available ( 2 and $5 \mathrm{~cm}^{2}$ ) and these were used in various combinations so that larger areas of skin 
TABLE 1. Details of patients

\begin{tabular}{|c|c|c|c|c|c|c|}
\hline \multirow[b]{2}{*}{ Patient } & \multirow[b]{2}{*}{$\begin{array}{l}\text { Age } \\
(\mathrm{yr})\end{array}$} & \multicolumn{3}{|c|}{ Duration in years of } & \multirow[b]{2}{*}{$\begin{array}{c}\text { Levodopa } \\
\text { dose (mg/day) }\end{array}$} & \multirow[b]{2}{*}{$\begin{array}{c}\text { Other } \\
\text { medication }\end{array}$} \\
\hline & & $\begin{array}{l}\text { Parkinson's } \\
\text { disease }\end{array}$ & $\begin{array}{c}\text { Levodopa } \\
\text { therapy }\end{array}$ & $\begin{array}{c}\text { On-off } \\
\text { syndrome }\end{array}$ & & \\
\hline 1 & 45 & 12 & 12 & 9 & 950 & $\begin{array}{l}\text { Lithium } \\
\text { orphenadrine }\end{array}$ \\
\hline 2 & 39 & 8 & 8 & 4 & 950 & $\begin{array}{l}\text { Benzhexol } \\
\text { amantadine }\end{array}$ \\
\hline 3 & 54 & 22 & 17 & 9 & 2100 & $\begin{array}{l}\text { Lithium } \\
\text { clomipramine }\end{array}$ \\
\hline 4 & 45 & 9 & 6 & 5 & 1200 & $\begin{array}{l}\text { Benzhexol } \\
\text { trimipramine }\end{array}$ \\
\hline
\end{tabular}

${ }^{a}$ With a peripheral decarboxylase inhibitor.

were covered on subsequent days. The areas used were $2,4,7,10,15$, and $20 \mathrm{~cm}^{2}$. The patches were applied to a hairless area of chest wall skin (usually in the subclavicular or submammary regions) and they were stuck down with Microfoam tape (3M Company) so as to maintain close skin contact. Before patch application the patients washed and dried themselves gently with a towel, avoiding any degree of skin abrasion or trauma. They were asked not to use body lotions or talcum powder. Whenever possible patches were not applied to an area of skin that had been used on a previous day.

Patient 1:

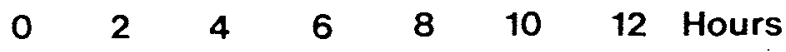

Patient 2:

Levodopa atone

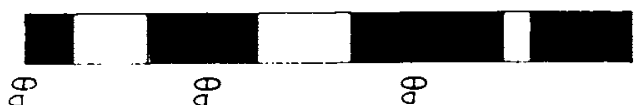

Levodopa alone

ब

里

8

Levodopa with PHNO

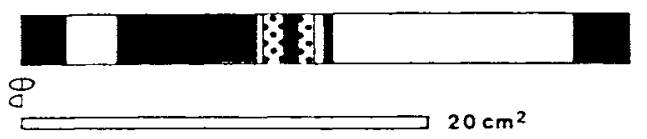

$20 \mathrm{~cm}^{2}$

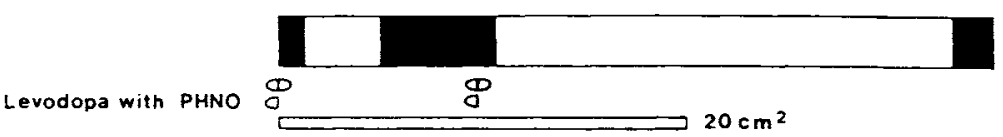

Key :
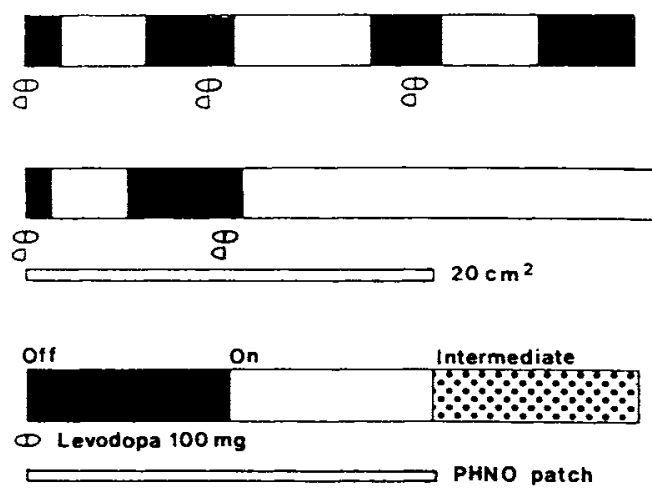

FIG. 1. The "on-off' charts for patients 1 and 2 comparing the effect of levodopa alone (normal response) and levodopa plus + PHNO $\left(20-\mathrm{cm}^{2}\right.$ patch). Hour 0 was $\sim 8: 00$ a.m. 
The first two patients had patches applied in the early morning ( 8:00 a.m.) and these remained in place for up to $8 \mathrm{~h}$. The second two patients had patches applied in the evening ( 8:00 p.m.) and kept in place for up to $24 \mathrm{~h}$. Patches were applied on alternate days so that there was always a $24-h$ washout period between doses. The patients received oral domperidone $(60 \mathrm{mg} /$ day) throughout the study.

On each test day the patient took no antiparkinson medication after midnight so that they would expect to be "off" on waking the next morning. The patients were monitored by bedside observation, which allowed an accurate on-off chart to be completed. This method of assessment is described in more detail elsewhere (13). The period of observation started at $\sim 8: 00 \mathrm{a} . \mathrm{m}$., which corresponded to hour 0 for patients 1 and 2 , and to hour 12 for patients 3 and 4 . If no effect from + PHNO was detected (i.e., if the patient did not switch "on") then a single dose of levodopa was given and repeated if necessary every $4 \mathrm{~h}$. During each study period blood samples were drawn for estimation of + PHNO concentration. At the end of each study period the patches were removed and the skin was examined for signs of local irritation or allergy.

\section{+PHNO Assay}

Measurement of + PHNO plasma concentration was carried out using a radioimmunoassay, complete details of which will be published elsewhere. Antibodies

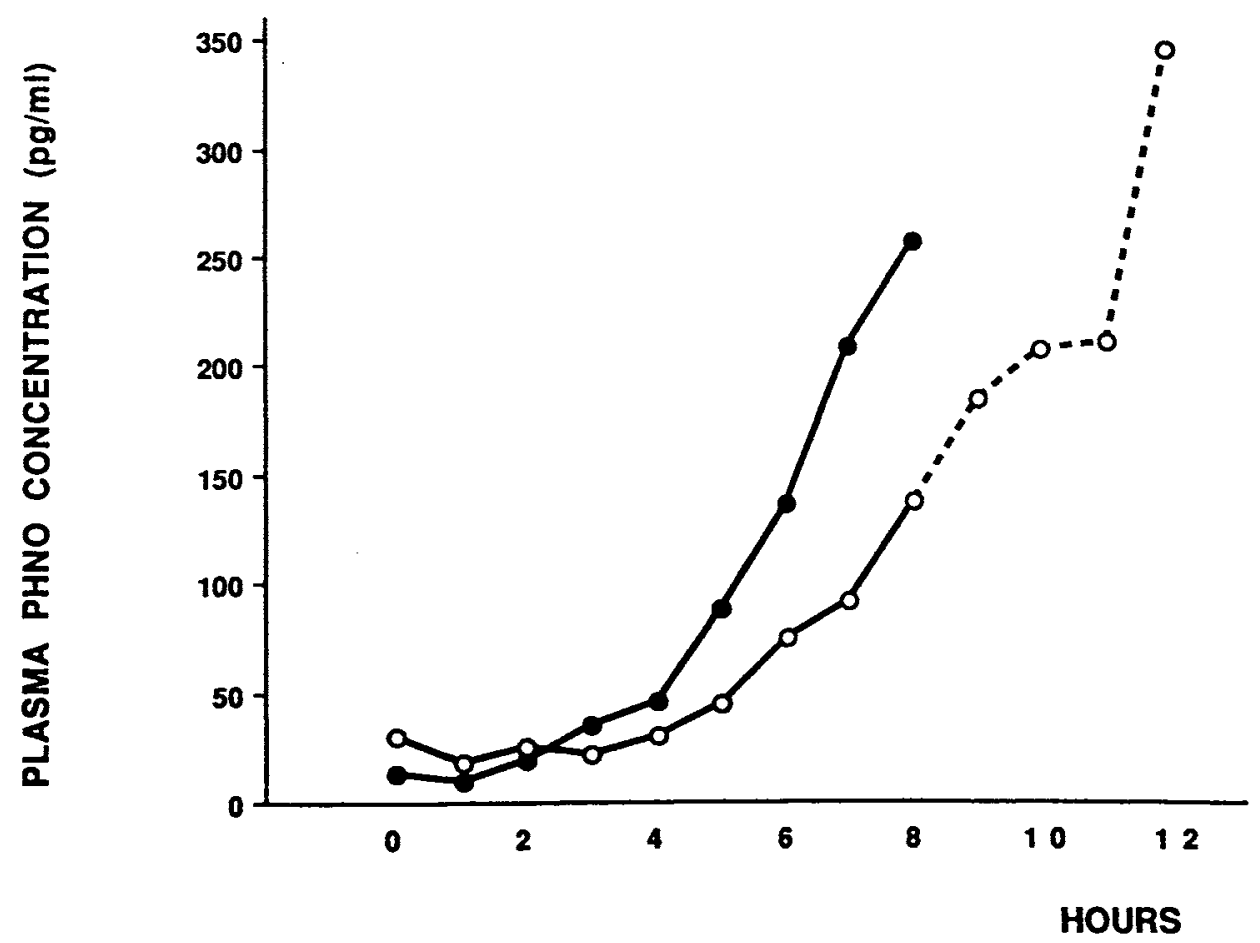

FIG. 2. Plasma concentrations of + PHNO in patients 1 and 2 after the application of $20-\mathrm{cm}^{2}$ patches applied at hour 0 and removed at hour $8 .--=$ Patient $1,-0-=$ patient $2,-0 \cdots=$ patient 2 after patch removal. 
were raised using 9-O-carboxypropyl-PHNO coupled to bovine serum albumin as the immunogen. The radioligand was 8 -iodo(I-125)-PHNO. A double antibody radioimmunoassay was established with a $1-\mathrm{ml}$ total volume using antisera at a final dilution of up to 5 million, and a sensitivity of $0.3 \mathrm{pg}$ per tube. Fifty percent inhibition of ligand binding occurred at $\sim 3 \mathrm{pg}$ per tube. Biological samples were extracted at $\mathrm{pH} 9.2$ with methyl $t$-butyl ether, and the drug was backextracted into $0.01 \mathrm{~N}$ hydrochloric acid. Up to $0.2 \mathrm{ml}$ of plasma was assayed, yielding a sensitivity of $\sim 2$ pg PHNO per milliliter plasma. Recoveries were $>90 \%$ and independent of concentration.

\section{RESULTS}

Patients 1 and 2 were monitored for between 4 and $8 \mathrm{~h}$ after application of patches of each size. In neither case was any clear effect seen until the maximum area of $20 \mathrm{~cm}^{2}$ was used. With this dose an effect was found that started between 4 and $6 \mathrm{~h}$ after patch application, and this is illustrated by the relevant on-off charts (Fig. 1). Without + PHNO, a standard dose of levodopa (150 mg levodopa with carbidopa as Sinemet 110) produced a response (i.e. the patient turned "on") that lasted for between 35 and $160 \mathrm{~min}$ (mean $103 \mathrm{~min}$ ). The onset and termination of this response was abrupt and therefore its duration could be recorded with accuracy. For each patient this response can be compared with the effect of levodopa in combination with $20 \mathrm{~cm}^{2}+$ PHNO skin patches. In both patients an initial dose of levodopa gave rise to the normal length of "on" response. Patient 1 switched "on" again spontaneously at $4 \frac{1}{2} \mathrm{~h}$ (without further levodopa), and after a number of "on-off" fluctuations stayed "on" for $6 \mathrm{~h}$ onwards. Brief
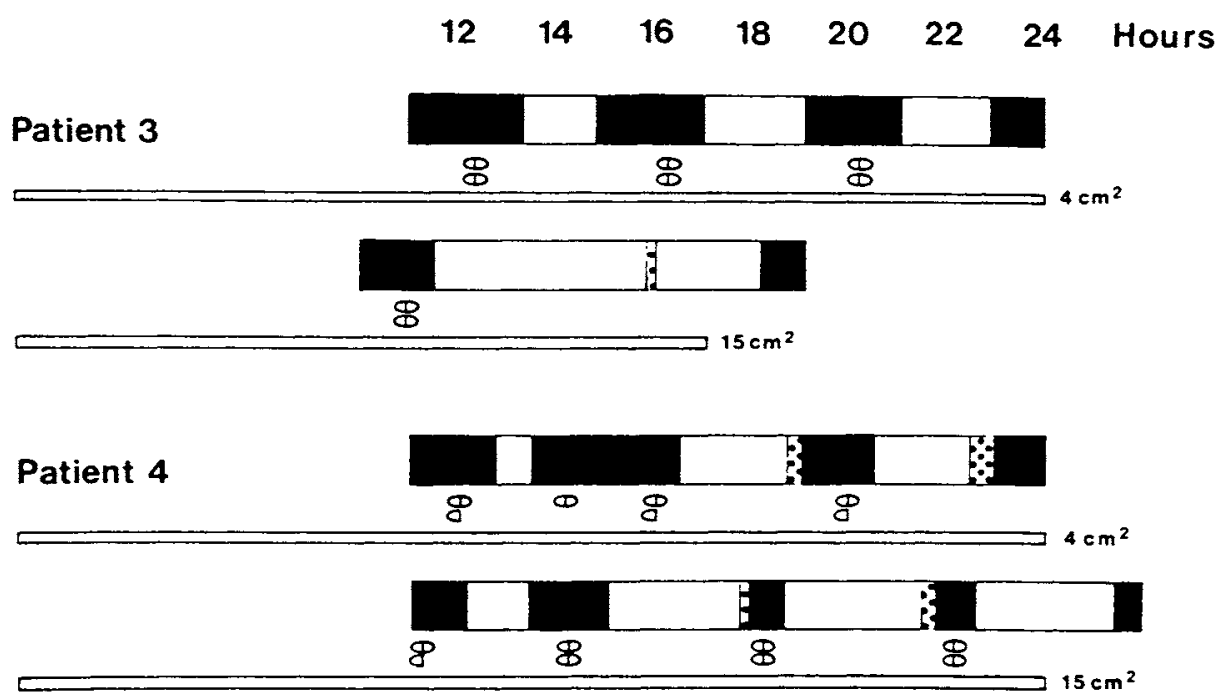

FIG. 3. The "on-off" charts for patients 3 and 4 comparing the effect of 4 and $15-\mathrm{cm}^{2}$ skin patches. Hour 12 was $\sim 8: 00$ a.m. (Key as for Figure 1.) 
periods could not be classified as either fully "on" or fully "off" and these were called "intermediate." Patient 2 required a second dose of levodopa, but its effect was prolonged far beyond what would normally have been expected. After the patches were removed, the response continued in both patients without further levodopa (in patient 1 for a further $3 \mathrm{~h}$ and in patient 2 for just more than $5 \mathrm{~h}$ ). The + PHNO plasma concentrations corresponding to these two doses are shown in Fig. 2.

Patients 3 and 4 had patches applied at night and were monitored in the same way during the following day. None of the skin patches (from 4 to $20 \mathrm{~cm}^{2}$ ) turned these patients "on" when they awoke, so they were given levodopa. The patches of $4-\mathrm{cm}^{2}$ size had little effect, but larger areas gave rise to longer "on" periods in

PATIENT 3

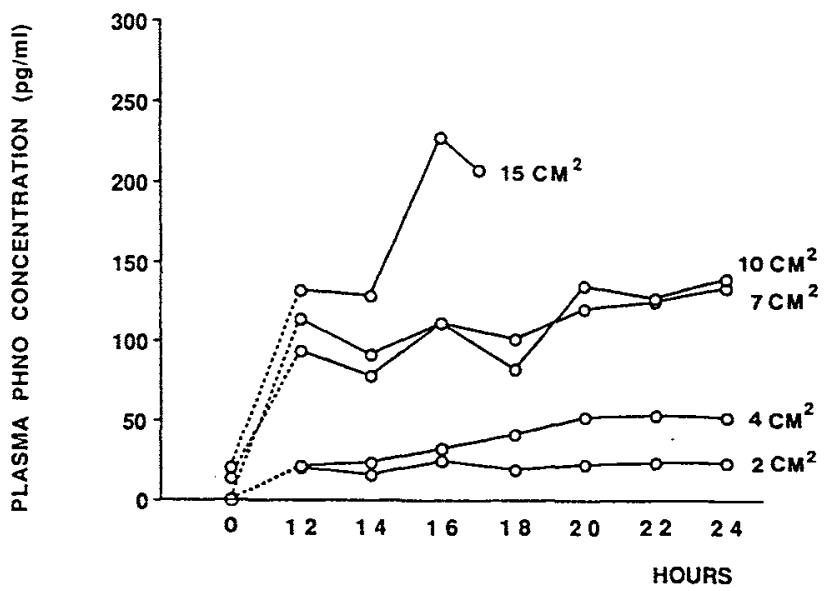

PATIENT 4

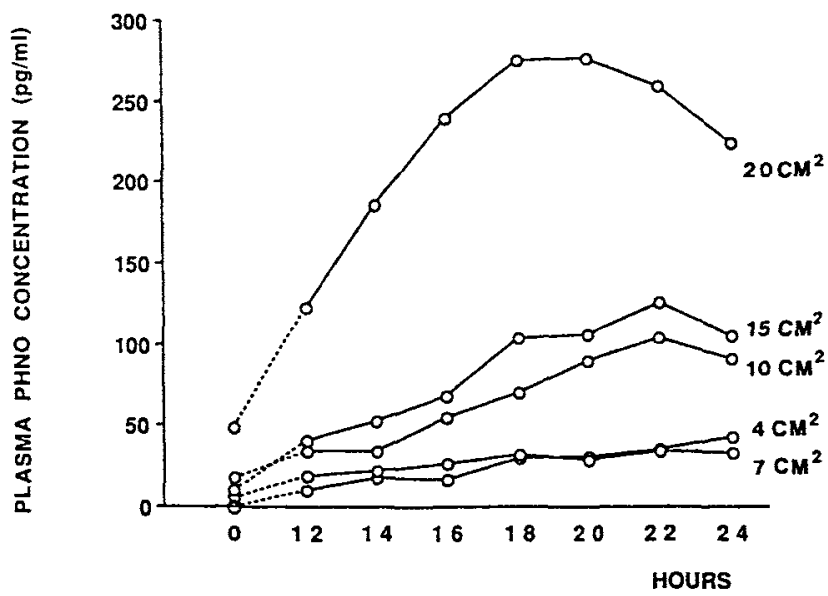

FIG. 4. Plasma concentrations of + PHNO achieved by increasing patch area in patients 3 and 4 . 
response to regular levodopa doses. Sample on-off charts (for 4 and $15 \mathrm{~cm}^{2}$ ) illustrate this (Fig. 3). With the $15-\mathrm{cm}^{2}$ patch, patient 3 developed excessive chorea that became distressing at $16 \mathrm{~h}$ and so the patches were removed early. This patient did not subsequently receive $20 \mathrm{~cm}^{2}$. The + PHNO plasma concentrations for patients 3 and 4 increased with larger patch area and they appeared to approach a steady state between 20 and $24 \mathrm{~h}$ (Fig. 4). A mean of the last three levels obtained on each day (e.g., at 20,22 , and 24 h) provides an approximate "steady state" level and this appears to be governed by the total patch area used (Fig. 5). Similarly, the clinical response (calculated as length of time spent "on" per $100 \mathrm{mg}$ of levodopa taken during the time of observation) can be related to the "steady state" + PHNO plasma concentration as already defined (Fig. 6). Regression lines have been calculated; for each patient the correlations between patch area and plasma concentration and between plasma concentration and clinical response were statistically significant (Figs. 5 and 6).

No serious side effects were identified. Two patients experienced excessive chorea with the largest patch area used and this was similar to that sometimes induced by taking too much levodopa. In patient 2 the dyskinesias were accompanied by anorexia and nausea and the intensity of these side effects reached a peak $\sim 2 \mathrm{~h}$ after the patches were removed. No skin irritation was observed.

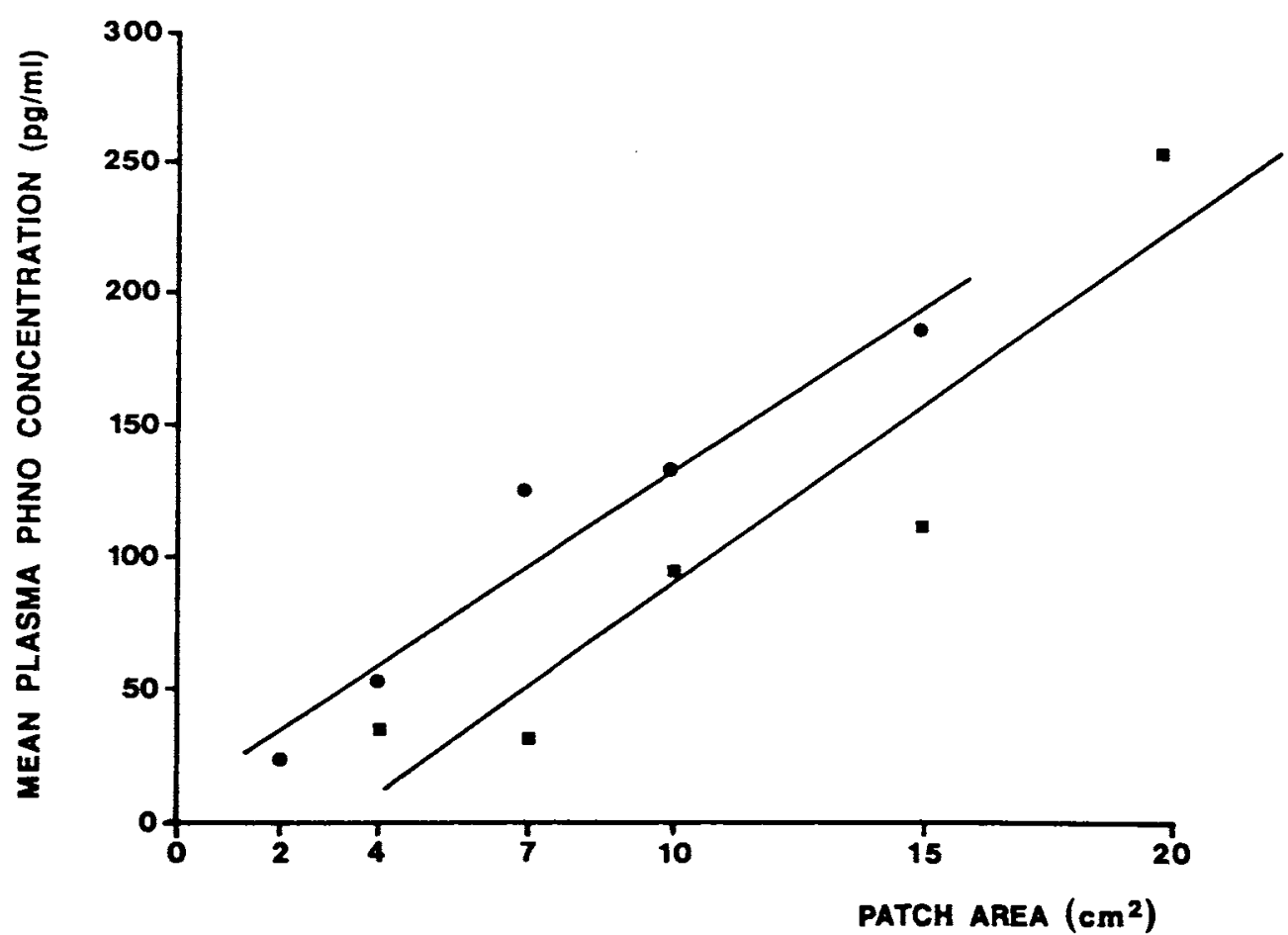

FIG. 5. The "steady state" plasma levels (mean of the last three values) is proportional to the area of patch used. Patient $3(O)$ : correlation coefficient $=0.968 ; t=6.70 ; d f=4 ; \mathrm{p}<0.01$. Patient 4 (目): correlation coefficient $=0.937 ; t=4.66 ; d f=4 ; p<0.05$. 


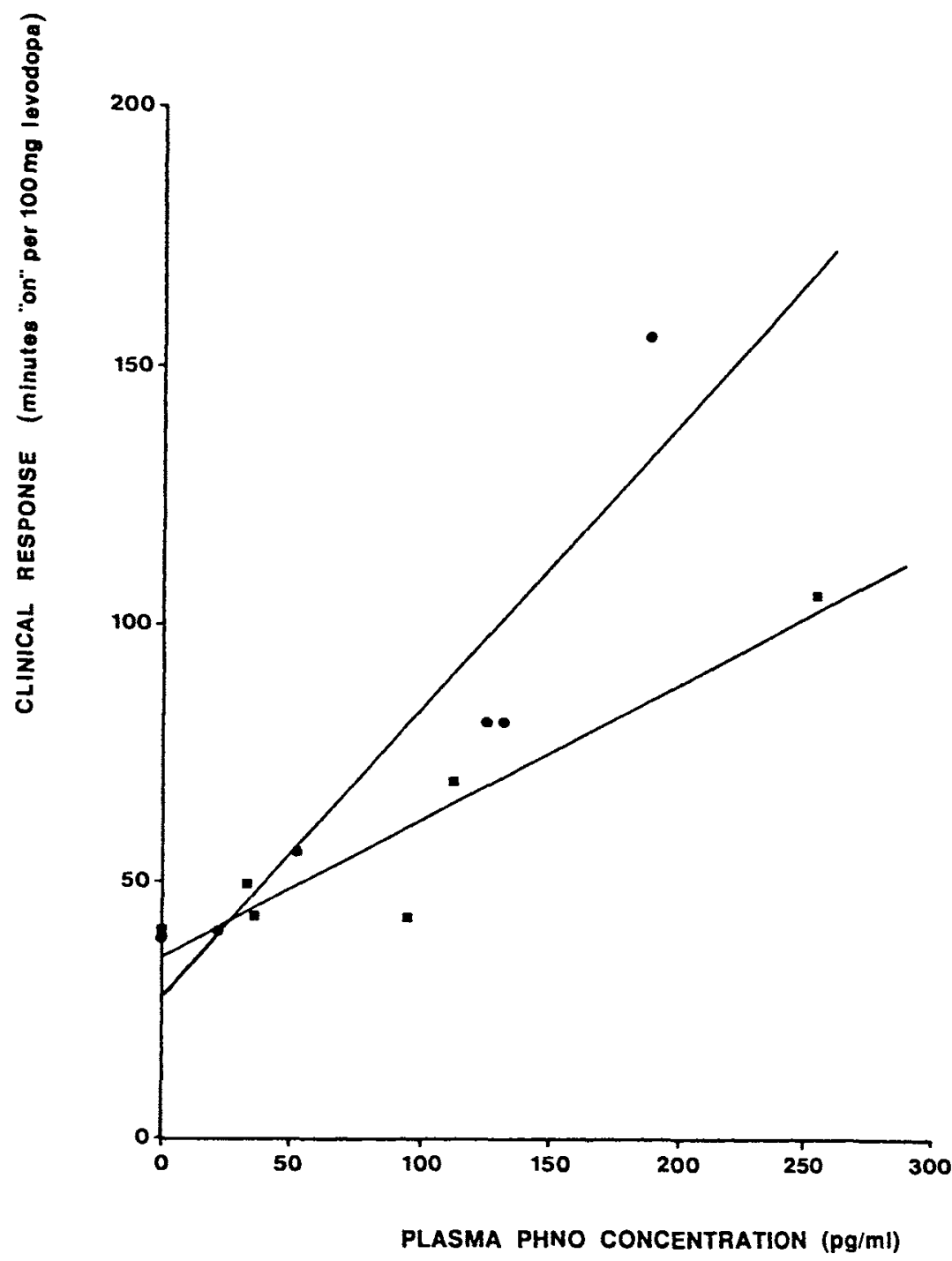

FIG. 6. The clinical response to levodopa (measured as minutes "on" per $100 \mathrm{mg}$ levodopa) is proportional to the "steady state" + PHNO concentration. Patient $3(0)$ : correlation coefficient $=$ $0.92 ; t=4.8 ; d f=4 ; \mathrm{p}<0.01$. Patient $4(\mathrm{~m})$ : correlation coefficient $=0.94 ; t=6.0 ; d f=4 ; \mathrm{p}<0.005$.

\section{DISCUSSION}

All four patients showed a response to + PHNO skin patches. In the first two patients, there was a clear difference between the response to levodopa alone and the response to levodopa with + PHNO $\left(20 \mathrm{~cm}^{2}\right)$. For both these patients the onset of action appeared to occur between 4 and $6 \mathrm{~h}$ after patch application. The plasma + PHNO levels corresponded with this, starting to rise $3-4 \mathrm{~h}$ after application and continuing to rise until the time of removal. The clinical response of 
both patients continued for several hours after removal and the side effects of patient 2 (chorea and nausea) reached maximum severity at that time. In this patient, plasma concentrations continued to rise for $4 \mathrm{~h}$ after patch removal (Fig. 2) although the unexpectedly large rise between hours 11 and 12 is unexplained.

The delay between patch application and rising plasma levels represents the time taken for + PHNO to permeate the epidermis and reach the dermal capillaries. The skin and subcutaneous tissues appear to act as an additional "drug reservoir" between the patch and the systemic circulation so that the patch must saturate this compartment before the drug can begin to enter the bloodstream. + PHNO retained in the skin continues to enter the circulation after the patches have been removed.

The data obtained from the first two patients show that it takes longer than $8 \mathrm{~h}$ for + PHNO from these patches to achieve steady-state plasma levels. This suggests that the patch size required to produce an effect in less than $8 \mathrm{~h}$ (i.e., $20 \mathrm{~cm}^{2}$ ) would be in danger of overdosing these patients if left in place for a much longer period. In light of these results it was decided to monitor the effects of all patch sizes for $24 \mathrm{~h}$ in patients 3 and 4 . No information was obtained from these two patients regarding the onset of + PHNO action. The plasma drug concentrations reached (or closely approached) a steady state between 18 and $24 \mathrm{~h}$ after patch application (Fig. 4). The final + PHNO levels were governed by the size of patch used and this confirms the notion that the area of skin covered represents the dose of drug administered.

In the present study we have shown that + PHNO and levodopa may be used successfully in combination because + PHNO lengthens the patients' response to individual levodopa doses. The degree of levodopa enhancement appears to be proportional to the + PHNO dose used and the plasma levels achieved. In these patients transdermal + PHNO did not produce a long continuous response without additional levodopa, although we have shown previously that oral + PHNO can switch patients "on" without levodopa (8).

In light of these results, we are hopeful that continuous transdermal delivery of + PHNO will become a useful technique in the treatment of parkinsonian "onoff' fluctuations.

\section{REFERENCES}

1. Marsden CD, Parkes JD. On-off effects in patients with Parkinson's disease on chronic levodopa therapy. Lancet 1976;1:292-6.

2. Fahn S. On-off phenomenon with levodopa therapy in parkinsonism. Neurology 1974;24:431-41.

3. Stibe CMH, Lees AJ, Stern GM. Subcutaneous infusion of apomorphine and lisuride in the treatment of parkinsonian on-off fluctuations. Lancet 1987;1:871.

4. Stibe CMH, Kempster PA, Lees AJ, Stern GM. Subcutaneous apomorphine in parkinsonian on-off oscillations. Lancet 1988;1:403-6.

5. Martin GE, Williams M, Pettibone DJ, Yarbrough GG, Clineschmidt BV, Jones JH. Pharmacologic profile of a novel potent directly-acting dopamine agonist, (+)-4-propyl-9. hydroxynaphthoxazine ((+)PHNO). J Pharmacol Exp Ther 1984;230:569-76.

6. Nomoto $M$, Stahl S, Jenner $P$, Marsden $C D$. Antiparkinsonian activity of $(+)$-PHNO in the MPTP-treated common marmoset. Movement Disorders 1987;2:37-45.

7. Stoessl AJ, Mak E, Calne DB. (+)-4-propyl-9-hydroxynaphthoxazine (PHNO), a new dopaminomimetic, in treatment of parkinsonism. Lancet 1985;2:1330-1. 
8. Grandas-Perez FJ, Jenner PG, Nomoto M, et al. (+)-4-propyl-9-hydroxynaphthoxazine in Parkinson's disease. Lancet 1986;1:906.

9. Koller W, Herbster G, Gordon J. PHNO, a novel dopamine agonist, in animal models of parkinsonism. Movement Disorders 1987;2:193-9.

10. Michaels AS, Chandrasekaran SK, Shaw JE. Drug permeation through human skin: theory and in vitro experimental measurement. AIChE J 1975;21:985-96.

11. Wepierre J, Marty JP. Percutaneous absorption of drugs. TIPS 1979;1:23-6.

12. Shaw JE, Urquhart J. Transdermal drug administration-a nuisance becomes an opportunity. $\mathrm{Br}$ Med J 1981;283:875-6.

13. Coleman RJ, Temlett JA, Quinn NP, Stahl SM, Marsden CD. A strategy for measuring the therapeutic properties of antiparkinsonian drugs in man. Clin Neuropharmacol (in press). 